\title{
Erros de medicação: intervenções adotadas pelos enfermeiros de educação continuada
}

\author{
Lilian Yukiko Kimura*, Marcio Antônio de Assis**
}

"Acadêmica do curso de enfermagem, Universidade de Mogi das Cruzes, participante do Programa Voluntário de Iniciação Cientifica (PVIC), **Enfermeiro, Doutorando em engenharia biomédica, docente da Universidade de Mogi das Cruzes (UMC)

\section{Resumo}

Objetivo: Este estudo teve como objetivo identificar as intervençôes realizadas pelos enfermeiros de educação continuada para prevenção de erros de medicação. Materiais e métodos: Foi realizada uma pesquisa survey exploratório-descritivo, de caráter quantitativo. A amostra foi constituída por enfermeiros que atuam em educação continuada na região do Alto Tiête/SP, através de amostragem por conveniência. Resultados: Os resultados demonstraram um cenário em que os enfermeiros realizam diversas intervençóes para prevenção de erros de medicação, porém há escassez no processo avaliativo dessas intervençóes, evidenciando a necessidade de aprofundamento desta temática pelos enfermeiros atuantes em educação continuada. Conclusão: A educação continuada atua na melhoria da qualidade na assistência à saúde através da sistematização do aprendizado, conscientização e mudanças geradas a partir de estratégias educacionais. Na prevenção de erros de medicação há diversos sistemas que visam a segurança do paciente na assistência à saúde. Neste contexto, a educação continuada desses profissionais proporciona condiçóes facilitadoras para que haja diminuição significativa de erros na medicação.

Palavras-chave: erros de medicação, enfermagem, educação continuada.

\section{Abstract}

\section{Medication errors: interventions adopted by nurses in continuing education}

Objective: This study aimed to identify the interventions performed by nurses who attend continuing education program for prevention of medication errors. Methods: We conducted an exploratory descriptive survey research, quantitative character. The sample consisted of nurses working in continuous education in the Alto Tietê/SP, through convenience sampling. Results: The results showed a scenario where nurses have done many interventions to detect and prevent medication errors. However, there are few such measures in the evaluation process, highlighting the need for deeper understanding of this issue by the nurses in continuing education. Conclusion: Continued education acts on health care quality improvement through learning systematization, raising health professionals conscience on the matter and educational strategies changing. In the prevention of medication errors there are several systems aimed at patient safety in health care. In this context, continuing education provides enabling conditions for a significant decrease in medication errors.

Key-words: medication errors, nursing, continuing education. 


\section{Resumen}

\section{Errores de medicación: Intervenciones adoptadas por el personal de enfermería en educación continua}

Objetivo: Este estudio tuvo como objetivo identificar las intervenciones realizadas por el personal de enfermería en educación continua para prevenir errores de medicación. Materialy métodos: Se ha realizado una investigación tipo survey exploratoria-descriptiva de carácter cuantitativo. La muestra fue compuesta de enfermeros que actúan en educación continua en la región del Alto Tiête - SP, a través del muestreo por conveniencia. Resultados: Los resultados muestran un escenario donde los enfermeros realizan diversas intervenciones para prevenir errores de medicación, sin embargo hay escasez en el proceso de evaluación de estas intervenciones, que evidencian la necesidad de estos profesionales, que actúan en educación continua, profundizar en esta temática. Conclusión: La educación continua actúa en la mejora de la calidad de atención de salud a través de la sistematización del aprendizaje, concientización de cambios a partir de estrategias educacionales. En la prevención de errores de medicación hay diversos sistemas que tienen por finalidad la seguridad del paciente en atención de salud. En este contexto, la educación continua del profesional de enfermería proporciona condiciones facilitadoras para reducir los errores de medicación.

Palabras-clave: errores de medicación, enfermería, educación continua.

\section{Introdução}

Atualmente a preocupação das instituições e profissionais de saúde vem crescendo em relação à segurança do paciente. Um estudo do National Center for Health Statistics, nos Estados Unidos da América (EUA), demonstrou as razóes de morte de pacientes em hospitais americanos e constatou que 44.000 foram causadas por eventos iatrogênicos, ultrapassando as mortes atribuídas a acidentes automobilísticos (43.458), câncer de mama (43.397) e AIDS (16.516) [1].

A segurança do paciente pode apresentar pontos críticos em quaisquer etapas do processo de hospitalizaçáo, principalmente em relação aos procedimentos que são realizados para atender à terapia necessária.

A administração de medicamentos é um procedimento multidisciplinar e de extrema responsabilidade do profissional de saúde que o executa, pois representa potenciais agravos e riscos à saúde do indivíduo [2]. Dessa forma, é importante ter em mente que falhas nesse processo podem ocorrer.

O erro na administração de medicamentos é definido como qualquer evento adverso evitável que pode resultar em danos ou prejuízos ao paciente e que pode ter sido gerado no processo de prescrição, dispensaçáo e/ou administração dos medicamentos, sendo possível ocorrer em qualquer uma dessas etapas do processo [3-5].

O custo anual para as instituições de saúde nos Estados Unidos por erros de medicação relacionados com morbidade e mortalidade é de aproximadamente US\$76,6 bilhôes [6].

De acordo com a American Society of Health-System Pharmacists (ASHP, 1993) é possível classificar e categorizar o erro de medicação conforme o tipo, causas, local da ocorrência, classificação terapêutica do medicamento e o resultado ocasionado no paciente [7].

Os erros mais frequentemente encontrados são de prescrição, omissão, horário, administração de medicamento nâo autorizado, dosagem incorreta, erro de apresentação, de preparo, técnica de administração inadequada, medicamentos deteriorizados e erro no monitoramento após a sua administração [8].

Uma das explicaçôes observadas para um grande número de erros é a ausência ou ineficácia de mecanismos, medidas e sistemas que diminuam a sua ocorrência ou que interceptem o erro antes de ser administrado ao paciente $[9,10]$. É importante lembrar que erros sempre vão ocorrer, porém minimizá-los requer, primeiramente, a aceitação de sua existência [9].

A Joint Commission on Accreditation of Healthcare Organizations, principal organização internacional que avalia a qualidade das instituiçôes prestadoras de serviço à saúde, propõe a inserção de medidas de qualidade e segurança do paciente, com a padronização de processos, instrumentos de notificação e indicadores de gerenciamento assistencial para minimizar e evitar os erros de medicação $[11,12]$. 
As estratégias e medidas para detecção e prevenção dos erros de medicação interferem diretamente na qualidade assistencial, sendo um importante indicador de qualidade com a mensuração através de avaliaçáo do desempenho da qualidade organizacional [10].

Existem várias estratégias que podem ser adotadas para prevenção de erros de medicação nas instituições hospitalares, como: normas específicas, regras, ações educativas, protocolos de padronização, notificaçóes de erros ocorridos, sistemas de medicaçôes, documentações, entre outras abordagens que visam à melhoria da qualidade da assistência à saúde $[6,13]$.

Em relação à equipe de enfermagem, cabe ressaltar que esses profissionais desempenham papel fundamental na prevenção de erros de medicação e possibilitam interceder em até $86 \%$ dos erros de medicação provindos da prescrição, transcrição e dispensação de medicamentos [14].

Sendo assim, torna-se importante manter uma equipe de enfermagem preparada para atender corretamente às necessidades da clientela que procura os serviços de saúde. Isso é possível por meio de orientaçóes e treinamentos que mantém uma equipe de enfermagem capacitada para prestar uma assistência à saúde segura.

Em uma instituição hospitalar isso se torna possível por meio de intervençôes do serviço de educação continuada (EC). Esse serviço busca um processo permanente que visa capacitar o conhecimento técnico-científico, propiciando novos conhecimentos e gerando atualizaçóes referentes à execução do trabalho inerentes a instituição hospitalar para os seus colaboradores $[15,16]$.

Este serviço é coordenado pelo profissional enfermeiro, que atua na melhoria da qualidade e segurança da assistência à saúde através da sistematização do aprendizado, conscientização, mudanças geradas a partir de estratégias de educação permanente, programas e atividades educacionais $[17,18]$.

É importante ressaltar que o enfermeiro da EC é responsável pela identificação das necessidades dos trabalhadores viabilizando condições que propiciem a prevenção de iatrogenias no cuidado prestado ao cliente [19]. Através de estratégias e de capacitação profissional por meio de educação contínua pode-se trabalhar mais efetivamente no que tange as causas sobre erros de medicação, minimizando assim os danos aos clientes e diminuindo os custos nas instituiçôes de saúde [10].
Sendo assim, é importante para as instituiçóes de saúde, profissionais e pacientes, que haja um trabalho voltado às intervençôes preventivas adotadas acerca dos erros de medicação, com a sensibilização dos profissionais de saúde, na tentativa de reduzir os potenciais erros existentes.

\section{Objetivo}

Identificar as intervençóes adotadas na minimização e prevenção de erros de medicação pelos enfermeiros atuantes em serviço de educação continuada da regiáo do Alto Tietê/SP.

\section{Material e métodos}

Trata-se de uma pesquisa survey exploratório-descritivo, de caráter quantitativo. A amostra foi constituída de treze enfermeiros que atuam em educação continuada na regiáo do Alto Tiête/SP, por amostragem de conveniência. Ressalta-se que, na região do Alto Tietê existem dezessete instituições hospitalares públicas e privadas, composta pelos municípios de Arujá, Santa Isabel, Mogi das Cruzes, Guararema, Biritiba Mirim, Suzano, Salesópolis, Poá e Ferraz de Vasconcelos.

Para a coleta dos dados foi utilizado um questionário semiestruturado elaborado pelos autores, composto pela caracterizaçáo do profissional e questóes abertas e fechadas direcionadas as intervençóes adotadas para prevenção de erros de medicações pelos enfermeiros do serviço de educação continuada. Realizou-se contato prévio com os particpantes, sendo agendado um dia específico, conforme a disponibilidade de cada um, para a aplicação do questionário.

O período de entrevistas foi entre os meses de agosto a outubro de 2010. Durante o período de coleta de dados, foi averiguado que em duas instituiçóes hospitalares não havia enfermeiro exclusivo de educação continuada para a realização da coleta de dados; e dois profissionais se recusaram a participar do estudo.

Após o esclarecimento de dúvidas referente à pesquisa e os participantes estarem cientes e de acordo em participar, os mesmos assinaram o Termo de Consentimento Livre e Esclarecido - TCLE, conforme as normas da Resolução 196/96. O projeto de pesquisa foi aprovado pelo Comitê de Ética em Pesquisa da Universidade de Mogi das Cruzes sob o número 0007.0.237.000-10. 
Os dados obtidos foram analisados quantitativamente, agrupando-os por semelhança de conteúdo e posteriormente apresentados em gráficos e tabelas por meio de frequências absolutas e relativas $(\%)$.

\section{Resultados e discussão}

\section{Dados demográficos}

As características da amostragem obtidas foram descritas na Tabela I.

Como podemos observar, houve predominância do sexo feminino $(69,23 \%)$, com tempo de formação acadêmica em média de 9,73 anos, sendo $61,54 \%$ dos entrevistados com pós-graduaçáo lato senso concluído principalmente nos cursos de administraçáo hospitalar e enfermagem em terapia intensiva, e no item pós-graduação stricto senso nenhum dos participantes possui esta formação. Pode-se dizer que a qualificaçáo profissional foi satisfatória, considerando que apenas 38,46\% não tinham uma pós-graduação em sua formação educacional, porém, notou-se que nenhum dos enfermeiros atuantes possui especialização relacionada à educação continuada (EC). Esta mesma característica foi observada num estudo com um grupo de enfermeiros de EC de uma instituição pública; percebeu-se que os enfermeiros tinham um conhecimento restrito em relação ao serviço de EC, setor de sua responsabilidade [20].

Quanto ao tempo de atuação na área, verificou-se que a média é de aproximadamente 3,41 anos, com predominancia de 69,23\% em instituição hospitalar pública, tendo em média 7,54 horas diárias nas atividades de EC.

\section{Intervençóes para prevenção de erros de medicação}

Após a caracterização da amostra, foram identificadas as intervençôes que os enfermeiros atuantes na EC adotam para a minimização e prevenção de erros de medicação, sendo assinalado pelos participantes as açóes que são utilizadas na sua prática profissional, descritas no Gráfico 1.

Gráfico 1 - Intervenções realizadas para prevenir os erros de medicação. Região do Alto Tietê/SP - 2010.

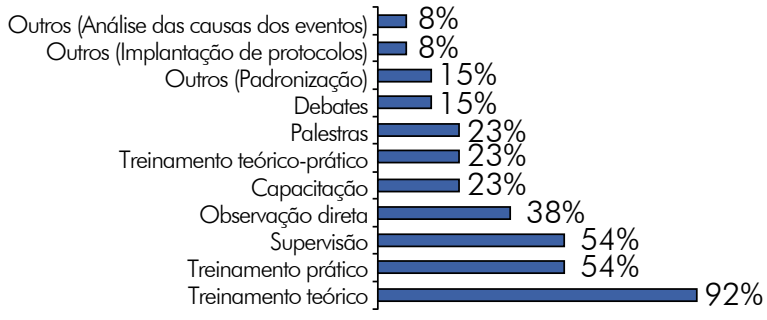

Podemos observar que os profissionais utilizam em média dois ou mais intervençóes para prevenção de erros de medicação, sendo os treinamentos

Tabela I - Características da amostra. Região Alto Tietê/SP - 2010.

\begin{tabular}{|c|c|c|c|c|c|c|}
\hline \multicolumn{7}{|c|}{ Características demográficas } \\
\hline & & $\mathrm{n}$ & $\%$ & & $\mathrm{n}$ & $\%$ \\
\hline Sexo & Masculino & 4 & $30,77 \%$ & Feminino & 9 & $69,23 \%$ \\
\hline Instituição Hospitalar & Pública & 9 & $69,23 \%$ & Privada & 4 & $30,77 \%$ \\
\hline \multirow[t]{6}{*}{ Pós-graduação Lato Sensu } & Sim & 8 & $61,54 \%$ & Não & 5 & $38,46 \%$ \\
\hline & Administração hospitalar & 3 & $18,75 \%$ & UTI & 3 & $18,75 \%$ \\
\hline & Licenciatura & 3 & $18,75 \%$ & Vigilância epid. & 1 & $6,25 \%$ \\
\hline & Nefrologia & 1 & $6,25 \%$ & Clínica Cirúrgica & 1 & $6,25 \%$ \\
\hline & Clínica médica & 1 & $6,25 \%$ & Pediatria & 1 & $6,25 \%$ \\
\hline & Gestão e auditoria & 1 & $6,25 \%$ & Cardiovascular & 1 & $6,25 \%$ \\
\hline \multicolumn{7}{|c|}{ Pós-graduação Stricto Sensu Nenhum participante possui. } \\
\hline Idade (anos) & Média (anos) & \multicolumn{2}{|c|}{37,08} & Desvio Padrão (anos) & \multicolumn{2}{|c|}{9,08} \\
\hline Tempo de formação & Média (anos) & \multicolumn{2}{|c|}{9,73} & Desvio Padrão (anos) & \multicolumn{2}{|c|}{7,117} \\
\hline Tempo de atuação & Média (anos) & \multicolumn{2}{|c|}{3,41} & Desvio Padrão (anos) & \multicolumn{2}{|c|}{4,16} \\
\hline Carga horária & Média (horas) & \multicolumn{2}{|c|}{7,54} & Desvio Padrão (horas) & \multicolumn{2}{|c|}{2,88} \\
\hline
\end{tabular}

${ }^{*} \mathrm{n}=$ número total da amostra 
teóricos (92\%) e práticos (54\%) as principais açóes educacionais realizadas. Este cenário reforça outros estudos feitos com enfermeiros de EC, que também identificaram estas atividades como as mais realizadas para assegurar o aprendizado contínuo dos profissionais de saúde [16,20].

Em seguida foi solicitado que os enfermeiros descrevessem em quais situaçóes eram utilizadas essas intervençôes assinaladas anteriormente, 28,57\% responderam que eram através de programas de aperfeiçoamento, atualização e/ou aprimoramento profissional, $28,57 \%$ na orientação inicial do colaborador recém-contratado, 19,05\% após a ocorrência de erro medicamentoso e $19,05 \%$ através de cronograma previamente estabelecido pelo serviço de EC. Ainda neste item foi citado que, para contribuir no aprimoramento e qualificação profissional, uma determinada instituiçáo hospitalar implementou um programa de incentivo institucional que possibilita a solicitação de folgas extras e reembolso financeiro para a realização de cursos e treinamentos externos aos colaboradores, assim propiciando mais uma alternativa para a capacitação da equipe de saúde (4,76\%).

Mediante os resultados o treinamento foi o mais citado como facilitador para as açóes educativas, visto que esta intervenção pode ser definida como um processo educacional que visa desenvolver habilidades, comportamentos, atitudes, conhecimentos e qualificar os recursos humanos para desempenhar suas tarefas específicas com excelência, através de uma estratégia que propicia soluçôes e promove conteúdos atualizados de acordo com os objetivos propostos pelo educador, na tentativa de prevenir potenciais danos ao paciente $[21,22]$.

Quanto à supervisão, esta ferramenta possibilita ao profissional obter com facilidade a identificação e de falhas causadas pela falta de conhecimento ou inexperiência dos colaboradores, sendo um dos motivos comuns para ocorrência de erros durante o cuidado assistencial [24].

Outro item questionado no estudo foi a frequência com que essas atividades educacionais são realizadas. Verificou-se que a maioria dos profissionais $(46,15 \%)$ não fazem um cronograma definido para executar suas açóes educacionais. Esta realidade dificulta o profissional no planejamento, desenvolvimento e no desempenho de suas atividades educacionais, contribuindo em resultados menos satisfatórios na minimização de ocorrência de erros de medicação para a segurança do paciente através do aprendizado contínuo [2,5,24,25].

Após a identificação das ações realizadas foi questionado sobre a existência de instrumentos específicos para o gerenciamento dos erros nas unidades de internação, $60 \%$ dos profissionais responderam que náo utilizam nenhum protocolo específico na sua rotina hospitalar. E dentre os profissionais que responderam que usam alguma ferramenta específica (40\%), 50\% relataram que os protocolos foram criados pela própria instituição hospitalar onde atuam e $50 \%$ responderam que o protocolo foi retirado de literatura específica. Os protocolos implementados citados foram: de procedimentos, de drogas de alto alerta, ficha de notificação de erros de medicaçáo e plano de aplicaçáo medicamentosa.

Considerando que a existencia de protocolos possibilita prevenir erros específicos, elimina dúvidas e possíveis falhas dos profissionais que executam a terapia medicamentosa, é uma importante ferramenta na prestaçáo do cuidado ao paciente [26]. Sendo assim, podemos observar que na realidade dos participantes é recomendada uma reflexão que permita melhorias no desenvolvimento do processo de qualidade, pois o processo de administração de medicamentos é bastante complexo, sendo relevante para uma prática com qualidade.

Quanto à existência de métodos ou instrumentos para detecção de erros de medicação, 60\% dos entrevistados responderam que não há nenhum método específico padronizado, e $40 \%$ responderam que têm instituído algum método ou instrumento como medida de detecção, sendo citadas: realização de busca ativa em prontuários através das anotaçóes de enfermagem, observação direta durante os procedimentos, utilização do diagrama de causa e efeito e ficha de notificação de erros envolvendo medicaçôes.

Os instrumentos de notificação permitem detectar as causas e falhas de ocorrências dos erros, elaborar índices, analisar as consequências e mensurar sua frequência na instituição hospitalar $[27,28]$. Possibilita averiguar as necessidades dos colaboradores a fim de planejar as açóes educacionais através da análise do erro.

Referente à notificação dos erros ocorridos, foi questionado se há incentivo para que as notificação sejam feitas, $60 \%$ dos enfermeiros de EC responderam que incentivam as notificaçóes nas unidades de internação, sendo $80 \%$ através dos enfermeiros líderes e $20 \%$ através de implementação de fichas específicas, com caráter não punitivo (100\%). 
Entretanto, quando questionados sobre a opiniáo da efetividade dessa atividade na sua realidade de trabalho, a maioria $(77,78 \%)$ respondeu que esta notificação não é feita de acordo com os erros ocorridos, tendo como justificativa deste cenário a insegurança dos profissionais de enfermagem frente a cultura punitiva.

Esta cultura de punir o profissional que errou foi fundamentada pelas medidas e providências administrativas que no passado eram realizadas, onde a ênfase na educação para prevenção destes erros e melhoria do sistema não era inserida [2,29]. Esta situação também pode estar relacionada por desconhecimento sobre o que esses erros podem acarretar ao paciente e ao profissional que cometeu tal erro; fato que interfere diretamente para um gerenciamento efetivo e de qualidade $[10,26]$. Afinal, a punição e a culpa geram dificuldades na identificação, análise e correção dos fatores que resultaram o erro medicamentoso, implicando consequentemente na dificuldade para reduzir as iatrogenias [30]. Sendo assim, estabelecer uma prática não punitiva aos profissionais na enfermagem é fundamental para interceptar e diminuir um maior número de erros na prática com a inserção da educação contínua e padronizaçáo dos processos medicamentosos na instituição hospitalar [31].

Quanto à avaliação dos resultados, constatou-se que a maioria dos participantes náo avalia suas intervençôes educacionais (80\%). A avaliação é uma das etapas do planejamento e tem a finalidade de verificar a eficácia das intervençóes realizadas, posicionando a educação continuada frente suas açóes executadas. Esta etapa é fundamental devido ao serviço ter um foco no processo contínuo, necessitando serem adequadas às necessidades dos colaboradores e da instituição hospitalar [32].

Esta escassez de mecanismos avaliativos dificulta o replanejamento e a reorganização das açóes educativas realizadas [18]. Tal situação impede uma análise sistêmica efetiva para detecção das vulnerabilidades existentes no sistema de medicação para que ocorra uma redução significativa dos erros [34].

Esta realidade também foi percebida em outros estudos relacionados à educação continuada, demonstrando a existência de dificuldades inerentes neste processo e a complexidade no serviço de educação continuada $[33,16]$.

Os profissionais que avaliam suas açóes educacionais especificaram a satisfação do cliente e do colaborador (10\%) e a avaliaçáo da eficácia do treinamento como os instrumentos avaliativos utilizados na rotina de trabalho (10\%).

A avaliação através da satisfação do cliente e do colaborador é um método que permite quantificar a satisfação do cliente e do colaborador através da identificação das necessidades e do desempenho do serviço prestado, com o foco na qualidade oferecida [35]. Quanto à avaliação da eficácia do treinamento, este instrumento avalia a reação do participante, a mudança de comportamento e a aprendizagem adquirida no treinamento [36]. Sendo uma diretriz da norma NBR ISO 10015:2001 para avaliar se o treinamento foi eficiente e eficaz, por gestáo de competências.

Os instrumentos de avaliação visam facilitar a compreensão de determinada situação, quantificar processos e possibilita uma visão da realidade para o gerenciamento e planejamento de açóes [37]. Cabe ressaltar que apenas dados coletados são inúteis se não gerar informaçóes no processo de decisão para a melhoria contínua da qualidade da assistência [10,37].

Referente às principais dificuldades enfrentadas na opinião dos participantes para minimização e prevençáo de erros de medicação foram descritas no Gráfico 2.

Gráfico 2 - Principais dificuldades enfrentadas pelos enfermeiros de EC. Região Alto Tietê/SP - 2010.

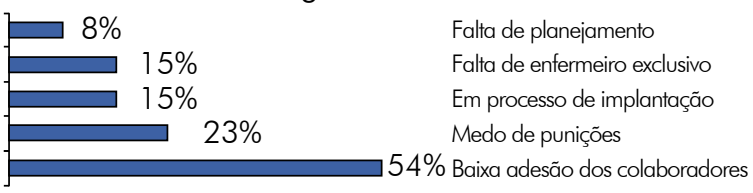

Percebe-se que $54 \%$ da amostra relatam a baixa adesão dos colaboradores nas atividades executadas pelo serviço de EC na sua realidade de trabalho, sendo o principal fator que impede a efetividade das açóes implementadas. Em seguida, novamente foi citado o medo de punição na ocorrência de erro medicamentoso pelos profissionais (23\%), evidenciando uma necessidade de mudança nos paradigmas frente às condutas tomadas pelos profissionais na ocorrência de erros de medicação e importância da contínua melhoria da prática do cuidar.

Referente à baixa adesão, alguns estudos observaram que esta característica pode ocorrer devido à sobrecarga de trabalho, treinamentos fora do horário de trabalho, ausência de subsídios para promover açóes educativas, fatores estruturais em consequência de ausência de parceria entre en- 
fermeiros assistenciais e da educação continuada, falta de planejamento e problemas administrativos comprometem o comparecimento dos trabalhadores nas atividades do serviço de educação continuada $[18,19,38$,$] . Outra situação que pode contribuir$ para esta situaçáo pode estar relacionada com o tempo planejado para a intervenção, desmotivação dos colaboradores e falta de divulgação da atividade oferecida [39].

Apesar da baixa efetividade das açóes realizadas, os enfermeiros de EC acreditam que os treinamentos, a orientação contínua e a orientação inicial realizados nas admissóes dos profissionais de enfermagem são as intervençôes mais efetivas na prevenção de erros de medicação. Estes dados reafirmam a efetividade da educaçáo contínua aos colaboradores para a prevenção de erros de medicação. Entretanto, ela deve estar vinculada a outras estratégias do sistema da terapia medicamentosa [2].

\section{Conclusão}

A prática de medidas educativas é um importante instrumento para o desenvolvimento do profissional em todos os sentidos, inclusive em relação à segurança na assistência prestada. Porém, percebe-se que esse fator não consegue ter um desfecho satisfatório se for conduzido de maneira isolada.

Este estudo viabilizou ampliar as reflexóes sobre a capacitaçáo e o processo de trabalho dentro de uma instituição hospitalar acerca da prevenção de erros de medicação, tendo em vista a educação contínua como uma ferramenta importante para a qualidade do cuidado prestado ao cliente.

Percebeu-se que os profissionais atuantes na EC acreditam que o treinamento e outras açóes educativas executadas são essenciais para diminuição e prevenção de iatrogenias medicamentosas. Portanto, é necessário que aliado ao serviço de educação continuada existam açóes voltadas para as mudanças de atitudes e paradigmas existentes nas instituiçôes hospitalares, fazendo com que o profissional tenha maior segurança em seus atos e seja acolhido adequadamente diante de uma situação iatrogênica.

\section{Referências}

1. Kohn LT, Corrigan JM, Donaldson MS. To err is human: building a safer health system. Committee on quality of health care in America, Institute of Medicine. Washington: National Academy Press; 2000.
2. Miasso AI, Silva AEBC, Cassiani SHB, Grou CR, Oliveira RC, Fakih FT. O processo de preparo e administração de medicamentos: identificação de problemas para propor melhorias e prevenir erros de medicaçáo. Rev Latinoam Enferm 2006;14(3):354-63.

3. National Coordinating Council for medications errors reporting and prevention. Ncc MERP taxonomy of medication errors. Rockville: Ncc MERP; 1998.

4. Rosa MB, Perini E. Erros de medicação: quem foi? Rev Assoc Med Bras 2003;49(3):335-41.

5. Bohomol E, Ramos LH. Erro de medicação: importância da notificação no gerenciamento da segurança do paciente. Rev Bras Enferm 2007;60(1):32-6.

6. Anderson JG, Jay SJ, Anderson M, Hunt TJ. Evaluating the capability of information technology to prevent adverse drug events: a computer simulation approach. J Am Med Inform Assoc 2002;9:479-90.

7. American Association of Hospital Pharmacists. ASHP. Guidelines on preventing medication errors in hospitals. Am J Hosp Pharm 1993;50:305-14.

8. Cousins DM. Defining medication errors: Medication use, a systems approach to reducing errors. Joint Comission 1998;39-56.

9. Carvalho M, Vieira AA. Medical errors in hospitalized patients. J Pediatric 2002;78(4):261-8.

10. Coimbra JAH. Prevençáo e detecçáo de erros de medicação. Ciência, Cuidado e Saúde 2006;5:142-8.

11. Nadzan DM. A system approach to medication use. In: Cousins DM, ed. Medication use: a system approach to reducing errors. Oakbrook Terrace (IL): Joint Commission on Accreditation of Healthcare Organizations; 1998. p.5-18.

12. Schneider PJ, Gift M. Measuring and monitoring the performance of the medication use system. In: Cousins DD. Medication Use: A Systems approach to reducing errors. Oakbrook Terrace (IL): Joint Commission on Accreditation of Healthcare Organizations. 1998. p.19-37.

13. Cassiani SHB. Administraçáo de medicamentos. São Paulo: EPU; 2000.

14. Leape LL, Berwick DM. Five years to err is human. What have we learned? JAMA 2005;293(19):2384-90.

15. Kurcgant P. Administração em enfermagem. São Paulo: EPU; 1991. p.147-53.

16. Koizumi MS, Koizumi MS, Kimura M, Miyadahira AMK, Cruz DALM. Educaçáo continuada da equipe de enfermagem nas UTIs do município de São Paulo. Rev Latinoam Enferm 1998; 6(3):33-41.

17. Ministério da Saúde. Secretaria de Gestáo do Trabalho e da Educação na Saúde. Departamento de Gestão da Educação na Saúde. Curso de facilitadores de educação permanente em saúde: unidade de aprendizagem trabalho e relaçôes na produção do cuidado. Brasília: MS; 2005.

18. Montanha D. Análise das atividades educativas de trabalhadores de enfermagem em um hospital de ensino: público participante, levantamento de necessidades e resultados esperados [dissertação]. São Paulo: Escola de Enfermagem, Universidade de Sáo Paulo; 2008.

19. Silva MF, Conceição FA, Leite MMJ. Educação continuada: um levantamento de necessidades da equipe de enfermagem. O mundo da saúde 2008;32(1):47-55. 
20. Davim RMB, Torres GV, Santos SR. Educação continuada em enfermagem: conhecimentos, atividades e barreiras encontradas em uma maternidade escola. Rev Latinoam Enferm 1999;7(5):43-49

21. Chiavenato I. Gestáo de pessoas. Rio de Janeiro: Elsevier; 2004. p.338-40.

22. Telles PCP Filho, Cassiani SHB. Administração de medicamentos: aquisiçâo de conhecimentos, atividades e barreiras encontradas em uma maternidade escola. Rev Latinoam Enferm 1999;7(5):43-49.

23. Carvalho VT, Cassiani SHB. Erros na medicaçáo e conseqüências para profissionais de enfermagem e clientes: um estudo exploratório. Rev Latinoam Enferm 2002;10(4):523-9.

24. Carvalho VT, Cassiani SHB, Chiericato C. Erros mais comuns e fatores de risco na administraçáo de medicamentos em unidades básicas de saúde. Rev Latinoam Enferm 1999; 7(5):67-75.

25. Oliveira RC, Camargo AEB, Cassiani, SHB. Estratégias para prevenção de erros na medicação no setor de emergência. Rev Bras Enferm 2005;58(4):399-404.

26. Bohomol E, Ramos LH. Erro de medicação: importância da notificação no gerenciamento da segurança do paciente. Rev Bras Enferm 2007;60(1):32-6.

27. Cassiani SHB, Miasso AI, Silva AEBC, Fakin FT, Oliveira RC. Aspectos gerais e número de etapas do sistema de medicação de quatro hospitais brasileiros. Rev Latinoam Enferm 2004;12(5):781-9.

28. Senst BL, Achusin LE, Genest RP, Cosentino LA, COrey C, Little JA, et al. Practical approach to determining costs and frequency of adverse drug events in a health care network. Am J Health Syst Pharm 2001;58(12):1126-32.
29. Teixeira TCA, Cassiani SHB. Análise de causa raiz: avaliação de erros de medicação em um hospital universitário. Rev Esc Enferm USP 2010;44(1):139-46.

30. Pedreira MLG, Marin HF. Patient safety initiatives in Brazil: a nursing perspective. Int J Med Inform 2004;73:563-7.

31. Womer RB, Tracy E, Soo-Hoo W, Bickert B, DiTaranto $\mathrm{S}$, Barnsteine JH. Multidisciplinary systems approach to chemotherapy safety: rebuilding processes and holding the gains. J Clin Oncol 2000;20(24):4705-12.

32. Silva GM, Seiffert OMLB. Educaçâo continuada em enfermagem: uma proposta metodológica. Rev Bras Enferm 2009;62(3):362-6.

33. Kristjanson IJ, Scanlan JM. Assessment of continuing nursing education needs: a literature review. J Contin Educ Nurs1992; 23(4):156-160.

34. Leape LL, Berwick DM, Bates DW. What practices will most improve safety? JAMA 2002;288(4):501-7.

35. Kotler P. Administração e Marketing. 5 ed. São Paulo: Atlas; 1998.

36. Associação Brasileira de Normas Técnicas. Norma ABNT NBR ISO 10015:2001. Diretrizes para treinamento; 2001.

37. Leão ER, Silva CPR, Alvarenga DC, Mendonça SHF. Qualidade em saúde e indicadores como ferramenta de gestão. São Paulo: Yendis; 2008.

38. Yamanaka TI, Pereira DG, Pedreira, MLG, Peterlini MAS. Redesenho de atividades da enfermagem para redução de erros de medicação em pediatria. Rev Bras Enferm 2007;60(2):190-196.

39. Hughes RG, Ortiz E. Medication errors: why they happen, and how they can be prevented. Am J Nurs 2005;105(3):14-24. 